Tropical Journal of Pharmaceutical Research December 2020; 19 (12): 2571-2576

ISSN: $1596-5996$ (print); 1596-9827 (electronic)

(C) Pharmacotherapy Group, Faculty of Pharmacy, University of Benin, Benin City, 300001 Nigeria.

Available online at http://www.tjpr.org

Original Research Article

http://dx.doi.org/10.4314/tjpr.v19i12.13

\title{
MiR-384 is associated with renal damage in lupus nephritis via regulation of TET3 expression
}

\author{
Jun Zhang ${ }^{1 *}$, Suli Lu², Ting Ding ${ }^{1}$, Haijia Zhao1, Dongxing Tang ${ }^{1 *}$ \\ ${ }^{1}$ Nephropathy and Rheumatism Department \& Hunan Province End Stage Renal Disease Clinical Medical Research Center, \\ Second Affiliated Hospital of University of South China, ${ }^{2}$ School of Medical, Hunan Polytechnic College of Environment and \\ Biology, Hengyang City, Hunan Province 421000, China
}

*For correspondence: Email: HYU0plhu9@163.com, HJKop145@163.com; Tel: +86-0734-8899699, +86-0734-8899699

Sent for review: 9 September 2020

Revised accepted: 23 November 2020

\begin{abstract}
Purpose: To investigate the correlations between miR-384 expression and renal damage in lupus nephritis (LN).

Methods: Lupus nephritis and normal tissues were collected during surgery. The relative miR-384 expression was evaluated by extracting RNA and performing quantitative real time PCR (qRT-PCR) assays. Expression of ten-eleven translocation (TET3) mRNA and protein were measured by qRT-PCR and western blotting, respectively. The 24-h urine protein, serum complement $C 3$, and serum creatinine were determined using commercial enzyme-linked immunosorbent assay (ELISA) kits. TargetScan and luciferase assays were used to validate the binding site for miR-384 and its target mRNA. Relationships among miR-384, TET3, and renal damage were analyzed by Spearman rank-order correlation coefficients.

Results: MiR-384 expression increased in LN tissues and was positively correlated with the activity index (Al) and chronicity index of LN, whereas miR-384 expression and serum complement C3 were negatively correlated. Positive correlations were observed between miR-384 expression and 24-h urine protein, serum creatinine, and systemic lupus erythematosus disease Al. TargetScan and luciferase assays indicated that the TET3 3'-UTR was the direct target of miR-384. MiR-384 upregulation inhibited TET3 mRNA and protein expression, and was negatively associated with renal damage in LN. Conclusion: MiR-384 upregulation contributes to renal damage in LN by targeting the 3'-UTR of TET3 $m R N A$, suggesting that miR-384 is a potential biomarker and therapeutic target in LN.
\end{abstract}

Keywords: MiR-384, Renal damage, Lupus nephritis, Ten-eleven translocation, TET3

This is an Open Access article that uses a funding model which does not charge readers or their institutions for access and distributed under the terms of the Creative Commons Attribution License (http://creativecommons.org/licenses/by/4.0) and the Budapest Open Access Initiative (http://www.budapestopenaccessinitiative.org/read), which permit unrestricted use, distribution, and reproduction in any medium, provided the original work is properly credited.

Tropical Journal of Pharmaceutical Research is indexed by Science Citation Index (SciSearch), Scopus, International Pharmaceutical Abstract, Chemical Abstracts, Embase, Index Copernicus, EBSCO, African Index Medicus, JournalSeek, Journal Citation Reports/Science Edition, Directory of Open Access Journals (DOAJ), African Journal Online, Bioline International, Open-J-Gate and Pharmacy Abstracts

\section{INTRODUCTION}

Systemic lupus erythematosus (SLE) is a chronic, systemic autoimmune disease that involves many organs, including the kidney [1]. Most patients with SLE develop lupus nephritis (LN) within 5 years [1]. Current therapeutic approaches for LN primarily use immunesuppressive drugs and anti-inflammatory medications [1]. However, the adverse effects of these medications include infection, infertility, and metabolic disturbances, which severely affect the quality of life of $L N$ patients [1]. Therefore, new therapeutic strategies should be 
developed with improved long-term efficacies. Recent studies found that DNA methylation is involved in the pathogenesis of LN [2]. Inhibition of DNA methylation suppressed the autoreactivity of $\mathrm{T}$ cells in $\mathrm{CD} 4^{+}$cells from lupus patients [3], indicating that modification of aberrant DNA methylation in LN may be a new therapeutic strategy.

Small noncoding microRNAs (miRNAs) have inhibitory effects on protein translation by directly targeting the 3'-untranslated region (3'-UTR) of mRNAs [4]. Many studies revealed that miRNAs were involved in the regulation of innate and adaptive immune responses [4]. For example, 66 aberrant miRNAs were identified using miRNA microarray chip analysis of renal biopsies of LN patients [5]. MiR-183 slowed the progression of LN and prolonged the survival of LN mice by reducing the immune complex component level, downregulating urine protein level, and restoring the number of Treg and T17 cells by targeting mTOR [6]. The expression of miR-130b-3b was upregulated in early $\mathrm{LN}$, was positively correlated with 24-h proteinuria and renal chronicity index $(\mathrm{Cl})$, and facilitated renal damage in early $\mathrm{LN}$ by binding to the 3 '-UTR of ERBB2IP [7]. However, the effect of miR-384 on LN is still not clear. Therefore, this study investigated the correlation between miR-384 expression and renal damage in $\mathrm{LN}$ and explored its underlying mechanism. The results of this study are expected to provide a new therapeutic approach for LN.

\section{EXPERIMENTAL}

\section{Human kidney tissue samples}

Lupus tissue samples $(n=56)$ were collected from LN patients, and normal kidney tissue samples $(n=23)$ were collected from patients with renal tumor as the normal control group (Table 1). The kidney tissue samples were collected at the Second Affiliated Hospital of University of South China and approved by the Medical Ethics Committee of University of South China (approval no. 20190620), in accordance with the Declaration of Helsinki [8]. All clinical materials complied with informed consent from patients.

\section{Cell culture and transfection}

Human glomerular mesangial cells (HMCs) were purchased from Ningbo Mingzhou Biotechnology Company (Mingzhoubio, China). HMCs were cultured in high-glucose DMEM supplemented with $10 \%$ fetal bovine serum (FBS, Beyotime, China) and $1 \%$ penicillin-streptomycin (Invitrogen, USA) at $37^{\circ} \mathrm{C}$ in a $5 \% \mathrm{CO}_{2}$ atmosphere. A total of $1 \times 10^{6} /$ well of HMCs were seeded in 6-well plates. The miR-384 mimics, miR-384 inhibitor, and its negative control (NC inh) were dissolved in Nanopure water and mixed with Lipofectamine 2000 (Invitrogen, USA) to form a transfection complex (1 $\mathrm{ng} / \mu \mathrm{L})$ according to the manufacturer's instructions. Then, $200 \mu \mathrm{L}$ of transfection complex was added to the cultured cells with FBS-free medium for 8 h. FBS-free medium was replaced with normal medium supplemented with $10 \%$ FBS, and the transfected cells were cultured for another $48 \mathrm{~h}$ for further study.

\section{Luciferase reporter assay}

A total of $1 \times 10^{6}$ cells/well of HMCs were seeded in 6-well plates and cultured overnight. The 3'UTRs of ten-eleven translocation (TET3) with wild-type (TET3-wt) or mutant (TET3-mut) miR384 binding site sequences were cloned into pmirGLO vector. The HMCs were co-transfected with cloned pmirGLO vector. The miR-384 mimics or their negative controls were transfected as described above. Luciferase activity was measured with the Dual-Luciferase Reporter (DLR) Assay System (Promega, USA).

\section{RNA extraction and quantitative real time PCR (RT-qPCR) assay}

Total RNAs were extracted using the TaKaRa MiniBEST Universal RNA Extraction Kit (TaKaRa, China). The concentrations of extracted RNAs were measured using a NanoDrop Microvolume Spectrophotometer (ThermoFisher, USA) before reverse transcription. The QIAGEN Multiplex PCR Kit (QIAGEN, Germany) was used for reverse transcription to cDNA. The RNA expression level was measured using the SYBR Green Quantitative RT-qPCR Kit (Sigma-Aldrich, USA) and the StepOnePlus system (Applied Biosystems, USA). The primer sequences (Sigma-Aldrich, USA) are listed in Table 1.

Table 1: Primer sequences used in this study

\begin{tabular}{ll}
\hline Gene & Primer sequence \\
\hline$\beta-$ & Forward: 5'-AGCCTCGCCTTTGCCGA-3' \\
Actin & Reverse: 5'-CTGGTGCCTGGGGCG-3' \\
TET3 & Forward: 5'-GCGCGGCATGGTATGAA-3' \\
& Reverse: 5'- \\
& ACTCGAGGTAGTCAGGGCATTCT-3' \\
\hline
\end{tabular}

The relative miR-384 expression level was determined using miScript Primer Assay (QIAGEN, Germany) and normalized with U6 small RNA. Relative RNA expression was calculated using $2^{-\Delta \Delta C t}$ method.

Trop J Pharm Res, December 2020; 19(12): 2572 


\section{Western blotting}

Total protein was extracted using RIPA buffer (Abcam, UK), and the protein concentration was measured using the Bradford Protein Assay Kit (Beyotime, China). Then, $5 \mu \mathrm{g}$ of protein was loaded and electrophoresed on a $15 \%$ polyacrylamide gel (SDS-PAGE). Proteins were transferred from SDS-PAGE to PVDF membranes (Merck KGaA, Germany). The PVDF membranes were blocked with Blocker BSA buffer (Thermo Fisher, USA), and then incubated with TET3 primary antibodies (CST\#85016, 1:500 dilution) and $\beta$-actin (CST\#4967, 1:5000 dilution) at $4{ }^{\circ} \mathrm{C}$ overnight. Horseradish peroxidase (HRP)-linked anti-rabbit lgG (CST\#7074, 1:2000 dilution) was used to probe target proteins. BeyoECL Moon (Beyotime, China) was used to detect the protein blot signals.

\section{ELISA assay}

Urine and blood samples were collected one day before renal biopsy and stored at $-80{ }^{\circ} \mathrm{C}$. After 24-h, urinary protein, serum creatinine, and serum complement C3 were measured using the Human Urine Protein ELISA Kit, Human SCreatinine ELISA Kit, and Human Complement C3 ELISA Kit (MyBioSource, Canada), respectively, according to the manufacturer's instructions.

Measurement of the activity index (AI) and chronicity index $(\mathrm{Cl})$ of $\mathrm{LN}$ and SLE disease activity

The $\mathrm{Al}$ and $\mathrm{Cl}$ of $\mathrm{LN}$ were measured using the US National Institutes of Health $(\mathrm{NIH})$ scoring system [9]. The SLE disease activity was obtained using the Systemic Lupus Erythematosus Disease Activity Index (SLEDAI) [10].

\section{Statistical analysis}

The results were analyzed using GraphPad Prism 8.1.0 and are expressed as mean \pm SD. Student's $t$-test was computed to analyze statistical differences between two groups. $P<$ 0.05 was considered as statistically significant.

\section{RESULTS}

\section{MiR-384 was overexpressed in LN tissues}

MiR-384 was overexpressed in LN tissues compared to normal tissues (Figure $1 \mathrm{~A}$ ). The relationship between miR-384 expression and LN was analyzed using Spearman's rank- order correlation. The expression of miR-384 was positively correlated with $\mathrm{Al}$ in $\mathrm{LN}$ patients (Figure $1 \mathrm{~B}$ ). Lupus nephritis patients with high miR-384 expression displayed higher $\mathrm{Cl}$ scores than those with low miR-384 expression (Figure $1 \mathrm{C})$.

A

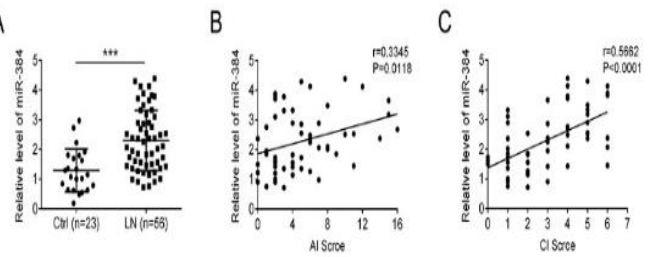

Figure 1: MiR-384 was upregulated in LN. (A) Expression of miR-384 was upregulated in $L N$ patients. (B) Al score was positively correlated with miR-384 expression. (C) $\mathrm{Cl}$ score was positively correlated with miR-384 expression. ${ }^{* * *} p<0.005$ versus control (Ctrl). Ctrl, normal tissues $(n=23)$; LN, lupus nephritis $(n=56)$; $\mathrm{Al}$, activity score of lupus nephritis; $\mathrm{Cl}$, chronicity index of lupus nephritis

\section{Expression of miR-384 was associated with renal damage in LN}

The expression of miR-384 was positively associated with 24-h urine protein in LN patients (Figure $2 \mathrm{~A}$ ), whereas the level of serum complement $\mathrm{C} 3$ was reduced in patients with miR-384 overexpression (Figure 2 B). Spearman's correlation coefficient between miR384 expression and serum creatinine was 0.5532 , with a $p$ value less than 0.0001 (Figure 3 C). A positive correlation was observed between miR-384 expression and SLEDAI (Figure 4 D).
A

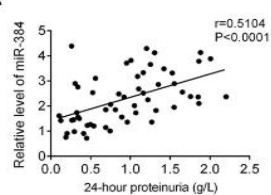

C

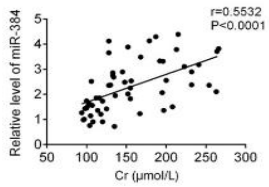

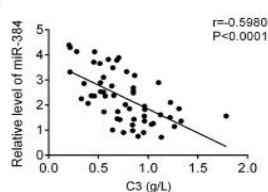

D

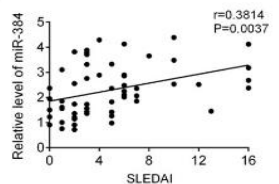

Figure 2: MiR-384 expression was positively correlated with renal damage in LN $(n=56)$. (A) The 24-h urine protein was positively correlated with miR384 expression. (B) Serum complement C3 was negatively correlated with miR-384 expression. (C) Serum creatinine was positively correlated with miR384 expression. (D) SLEDAI was positively correlated with miR-384 expression. C3, serum complement C3; $\mathrm{Cr}$, serum creatinine; SLEDAI, Systemic Lupus Erythematosus Disease Activity Index 
Upregulation of miR-384 repressed TET3 expression by targeting the $3^{\prime}$-UTR of TET3 mRNA

The molecular mechanism of miR-384 in LN was investigated using TargetScan (www.targetscan.org), which predicted that the 3'-UTR of TET3 was a direct target of miR-384 (Figure $3 \mathrm{~A}$ ). This prediction was validated by a luciferase activity assay, which showed that luciferase activity was significantly reduced in HMCs that were cotransfected with the TET3-wt vector and miR-384 mimics (Figure $3 \mathrm{~B}$ ). However, there were no significant differences between HMCs that were transfected with the TET3-mut vector and those transfected with miR384 mimics. To verify that miR-384 downregulates TET3 expression, miR-384 mimics, miR-384 inhibitors, and their negative controls were transfected into HMCs. The results indicated that miR-384 expression was significantly upregulated by miR-384 mimics and downregulated by miR-384 inhibitors (Figure $3 \mathrm{C}$, $p<0.001)$. Overexpression of miR-384 downregulated TET3, whereas inhibition of miR384 upregulated TET3 at both protein and mRNA levels (Figures $3 \mathrm{D}$ and $\mathrm{E}$ ).

A

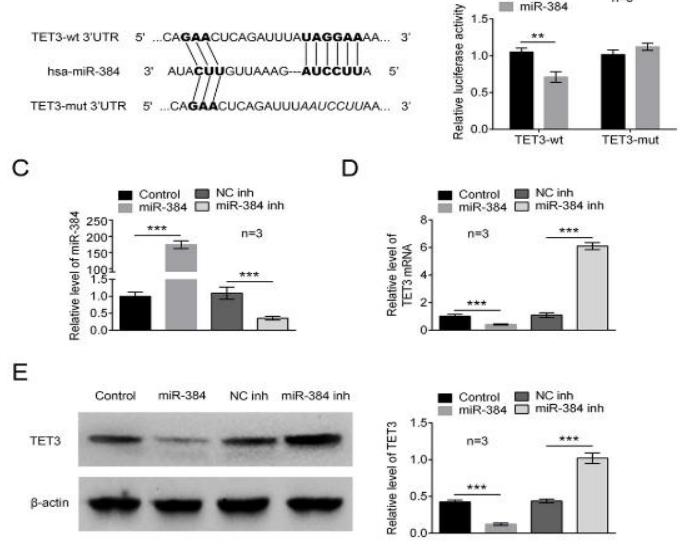

Figure 3: The 3'-UTR of TET3 was the direct target of miR-384. (A) TargetScan identified the binding site between the TET3 3'-UTR and miR-384. (B) Luciferase activity was reduced in HMCs cotransfected with TET3-wt vector and miR-384 mimics. (C) Expression of miR-384 was increased by miR-384 mimics and decreased by miR-384 inhibitors. (D) Overexpression of miR-384 downregulated TET3, whereas inhibition of miR-384 upregulated TET3 at the mRNA level. (E) Overexpression of miR-384 downregulated TET3, whereas inhibition of miR-384 upregulated TET3 at the protein level. All experiments were repeated three times. ${ }^{* *} p<0.01$ versus control or $\mathrm{NC}$ inh; ${ }^{* * *} p<0.005$ versus control or NC inh. Control, control mimics; NC inh, negative control of miR-384
Upregulation of miR-384 was correlated with downregulation of TET3

The RT-qPCR results demonstrated that the expression of TET3 mRNA was downregulated in LN tissues compared to normal tissues ( $p<$ 0.001 , Figure $4 \mathrm{~A}$ ). The expression of TET3 mRNA was negatively correlated with miR-384, with a Spearman's correlation coefficient of 0.5664 ( $p<0.0001$, Figure 4 B). Western blotting results indicated that the expression of TET3 protein was lower in LN tissues than in normal tissues (Figure $4 \mathrm{C}$ ).
A

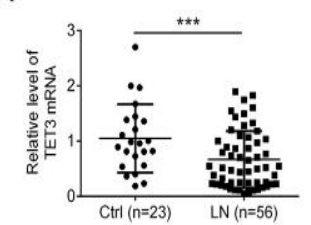

C

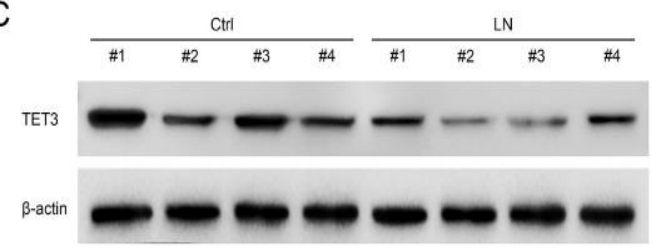

B

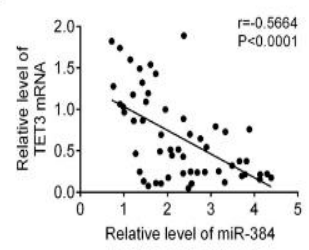

Figure 4: Expression of TET3 was downregulated in LN. (A) Expression of TET3 mRNA was downregulated in LN tissue. (B) Expression of TET3 mRNA was negatively correlated with miR-384 expression in LN tissue. (C) Expression of TET3 protein was downregulated in LN tissue. ${ }^{* * *} p<0.005$ versus control. Sample size: $\mathrm{LN}, n=56$; control, $n=$ 23

\section{TET3 expression was associated with renal damage in LN}

The expression of TET3 mRNA was significantly downregulated in patients with renal damage in LN (Figure 5). The reduced TET3 mRNA levels in $L N$ were associated with increases in $\mathrm{Al}, \mathrm{Cl}$, and SLEDAI scores (Figure $5 \mathrm{~A}, \mathrm{~B}$, and $\mathrm{F}$ ). The 24-h urine protein and serum creatinine levels were negatively correlated with the expression level of TET3 mRNA (Figure $5 \mathrm{C}$ and $\mathrm{E}$ ), whereas serum complement C3 was positively correlated with the expression level of TET3 mRNA (Figure 5 D).

\section{DISCUSSION}

Lupus nephritis is an aggressive glomerulonephritis with histopathological features such as deposition of immune complexes, leukopenia, hematuria, and proteinuria. 
A

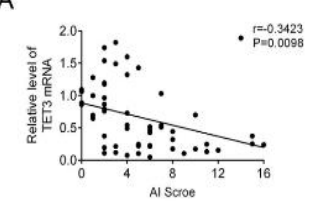

C

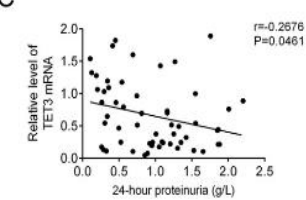

E

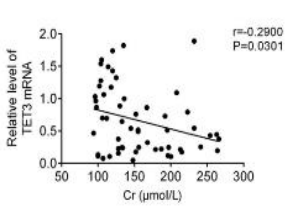

B

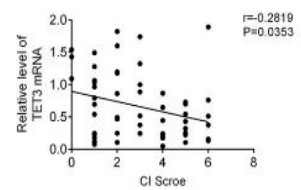

D

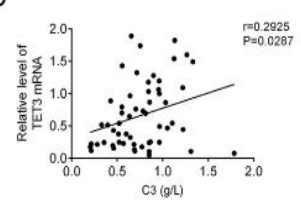

F

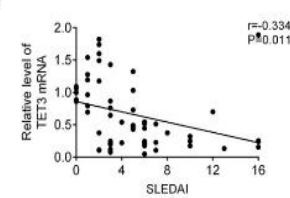

Figure 5: Expression of TET3 mRNA was negatively correlated with renal damage in LN $(n=56)$. (A) The Al score was negatively correlated with TET3 mRNA expression. (B) The $\mathrm{Cl}$ score was negatively correlated with TET3 mRNA expression. (C) The 24-h urine protein was negatively correlated with TET3 mRNA expression. (D) Serum complement C3 was positively correlated with TET3 mRNA expression. (E) Serum creatinine was negatively correlated with TET3 mRNA expression. (F) SLEDAI was negatively correlated with TET3 mRNA expression

Patients with LN will develop chronic kidney disease within 5 years, and 10-30\% of these patients ultimately develop end-stage renal disease (ESRD) [11]. A better understanding of LN pathogenesis and new therapeutic strategies are necessary to improve the long-term prognosis for LN patients. This study identified miR-384 overexpression in LN patients, along with higher 24-h proteinuria and serum creatinine levels. The $\mathrm{Al}$ and $\mathrm{Cl}$ scores of $\mathrm{LN}$ and the SLEDAI value were positively correlated with expression of miR-384. These results suggest that miR-384 contributes to renal damage and LN.

MiR-384 is involved in many pathological processes. For example, upregulation of miR384 in bone marrow mesenchymal stem cells inhibited osteogenic differentiation and accelerated senescence by targeting Gli2, thereby promoting age-related bone loss. MiR384 prevents ischemia-reperfusion-induced activation of cardiac fibrosis and TGF- $\beta$-induced cardiac fibroblasts through a multitargeted TGF$\beta /$ Wnt transactivation network [12]. The present study investigated the mechanism by which miR384 promoted renal damage in LN. TargetScan analysis predicted a complementary sequence

between miR-384 and the 3'-UTR of TET3. The results from a luciferase assay confirmed that the 3'-UTR of TET3 was the direct target of miR-384.

TET3 is a member of the TET family of proteins, which catalyze the demethylation of cytosine. Loss of DNA methylation is one of the key pathological mechanisms of SLE [13].

The present study showed that TET3 mRNA and protein expression was reduced in renal biopsies of LN patients; by contrast, a previous study reported that TET3 expression increased in SLE $\mathrm{CD}^{+} \mathrm{T}$ cells [14]. These results suggested that TET3 was differentially expressed in immune and non-immune tissues of SLE patients, which might have different roles in regulating TET3 gene expression.

Complement is a key immune system regulator and is involved in recruiting inflammatory cells, clearing immune complexes, mediating antimicrobial defense, and amplifying the humoral immune response [15]. Complement C3 participates in the activation of all three complement pathways [16]. Consistent with a previous study, our results showed that the serum complement C3 level was reduced in $\mathrm{LN}$ patients. Serum creatinine and urine protein are two biomarkers that are widely used for diagnosis and monitoring of renal function [17].

The present study observed that increases in serum creatinine and 24-h urine protein were associated with miR-384 upregulation, suggesting that miR-384 promoted renal damage in patients with LN. By contrast, TET3 expression in LN kidney tissue was inversely correlated with serum creatinine and 24-h proteinuria, suggesting a protective effect of TET3 on renal function in LN. Combined with the Spearman's correlation coefficients for $\mathrm{Al}, \mathrm{Cl}$, and SLEDAI, the results of this study indicate that miR-384 overexpression in $\mathrm{LN}$ exacerbated renal damage by directly inhibiting TET3.

\section{CONCLUSION}

This study observed miR-384 upregulation in LN tissues. Increases in 24-h proteinuria and serum creatinine levels were associated with miR-384 upregulation. MiR-384 targeting of TET3 resulted in renal damage in patients with $L N$, indicating that miR384/TET3 may be a novel therapeutic target for LN.

Trop J Pharm Res, December 2020; 19(12): 2575 


\section{DECLARATIONS}

\section{Conflict of interest}

No conflict of interest is associated with this work.

\section{Contribution of authors}

We declare that this work was done by the authors named in this article, and all liabilities pertaining to claims relating to the content of this article will be borne by the authors. JZ and DXT conceived and designed the experiments, SLL analyzed and interpreted the results of the experiments, and TD and HJZ performed the experiments. All authors read and approved the manuscript.

\section{Availability of data and materials}

All data generated or analyzed during this study were included in this published article.

\section{Open Access}

This is an Open Access article that uses a funding model which does not charge readers or their institutions for access and distributed under the terms of the Creative Commons Attribution License (http://creativecommons.org/licenses/by/ 4.0) and the Budapest Open Access Initiative (http://www.budapestopenaccessinitiative.org/rea d), which permit unrestricted use, distribution, and reproduction in any medium, provided the original work is properly credited.

\section{REFERENCES}

1. Anders H-J, Saxena R, Zhao M-h, Parodis I, Salmon JE, Mohan C. Lupus nephritis. Nat Rev Dis Primers 2020; 6(1): 7.

2. Chen SH, Lv QL, Hu L, Peng MJ, Wang GH, Sun B. DNA methylation alterations in the pathogenesis of lupus. Clin Exp Immunol 2017; 187(2): 185-192.

3. Richardson B. Effect of an inhibitor of DNA methylation on $T$ cells. II. 5-Azacytidine induces self-reactivity in antigen-specific T4+ cells. Hum Immunol 1986; 17(4): 456-470.

4. Liu X, Chen L, Liu Y, Zhang T. Tangeretin sensitises human lung cancer cells to TRAIL-induced apoptosis via ROS-JNK/ERK-CHOP pathway-mediated up-regulation of death receptor 5. Trop J Pharm Res 2017; 16(1): 1729.

5. Dai $Y$, Sui W, Lan $H$, Yan Q, Huang $H$, Huang $Y$. Comprehensive analysis of microRNA expression patterns in renal biopsies of lupus nephritis patients. Rheumatol Int 2009; 29(7): 749-754.

6. Li X, Luo F, Li J, Luo C. MiR-183 delivery attenuates murine lupus nephritis-related injuries via targeting mTOR. Scand J Immunol 2019; 90(5): e12810.

7. Wang $W$, Mou S, Wang L, Zhang $M$, Shao $X$, Fang $W, L u$ $R$, Qi C, Fan Z, Cao $Q$ et al. Up-regulation of Serum MiR-130b-3p Level is Associated with Renal Damage in Early Lupus Nephritis. Sci Rep 2015; 5: 12644-12644.

8. World Medical A. World Medical Association Declaration of Helsinki: Ethical Principles for Medical Research Involving Human Subjects. JAMA 2013; 310(20): 21912194.

9. Austin HA, Muenz LR, Joyce KM, Antonovych TA, Kullick ME, Klippel JH, Decker JL, Balow JE. Prognostic factors in lupus nephritis: Contribution of renal histologic data. Am J Med 1983; 75(3): 382-391.

10. Bombardier C, Gladman $D D$, Urowitz MB, Caron $D$, Chang $\mathrm{CH}$, Austin A, Bell A, Bloch DA, Corey $P N$, Decker $J L$ et al. Derivation of the sledai. A disease activity index for lupus patients. Arthritis Rheum 1992; 35(6): 630-640.

11. Alarcón GS. Multiethnic lupus cohorts: What have they taught us? Reumatología Clínica 2011; 7(1): 3-6.

12. Seo $H-H$, Lee $S$, Lee $C Y$, Lee J, Shin S, Song B-W, Kim I-K, Choi J-W, Lim S, Kim SW et al. Multipoint targeting of TGF- $\beta / W n t$ transactivation circuit with microRNA 384$5 p$ for cardiac fibrosis. Cell Death Differ 2019; 26(6): 1107-1123.

13. $W u H$, Zhao $M$, Tan $L, L u Q$. The key culprit in the pathogenesis of systemic lupus erythematosus: Aberrant DNA methylation. Autoimmun Rev 2016; 15(7): 684-689.

14. Zhao M, Wang J, Liao W, Li D, Li M, Wu H, Zhang $Y$, Gershwin ME, Lu Q. Increased 5-hydroxymethylcytosine in CD4+ $T$ cells in systemic lupus erythematosus. J Autoimmun 2016; 69: 64-73.

15. Popat RJ, Robson MG. Complement and Glomerular Diseases. Nephron Clin Pract 2014; 128(3-4): 238-242.

16. Vignesh $P$, Rawat $A$, Sharma $M$, Singh $S$. Complement in autoimmune diseases. Clin Chim Acta 2017; 465: 123130.

17. Sudhakaran S, Bottiglieri T, Tecson KM, Kluger AY, McCullough PA. Alteration of lipid metabolism in chronic kidney disease, the role of novel antihyperlipidemic agents, and future directions. Rev Cardiovasc Med 2018; 19(3): 77-88. 